\title{
FEATURE EXTRACTION BASED RETRIEVAL OF GEOGRAPHIC IMAGES
}

\author{
Mrs.S.Gandhimathi @ Usha ${ }^{1}$, M.Jeya Rani ${ }^{2}$, S.Sneha $^{3}$ and R.Kalaiselvi ${ }^{4}$ \\ ${ }^{1}$ Assistant Professor, ${ }^{2,3,4}$ B.E. Student \\ Department of Electronics and Communication Engineering \\ Velammal College of Engineering and Technology, Madurai.
}

\begin{abstract}
This project is to retrieve the similar geographic images from the dataset based on the features extracted. Retrieval is the process of collecting the relevant images from the dataset which contains more number of images. Initially the preprocessing step is performed in order to remove noise occurred in input image with the help of Gaussian filter. As the second step, Gray Level Co-occurrence Matrix (GLCM), Scale Invariant Feature Transform (SIFT), and Moment Invariant Feature algorithms are implemented to extract the features from the images. After this process, the relevant geographic images are retrieved from the dataset by using Euclidean distance. In this, the dataset consists of totally 40 images. From that the images which are all related to the input image are retrieved by using Euclidean distance. The approach of SIFT is to perform reliable recognition, it is important that the feature extracted from the training image be detectable even under changes in image scale, noise and illumination. The GLCM calculates how often a pixel with gray level value occurs. While the focus is on image retrieval, our project is effectively used in the applications such as detection and classification.
\end{abstract}

\section{KEYWORDS}

GRAY LEVEL CO-OCCURRENCE MATRIX (GLCM), SCALE INVARIANT FEATURE TRANSFORM (SIFT).

\section{INTRODUCTION:}

The need for efficient image retrieval has increased immensely in many applications such as biomedicine, military, and web image classification and searching. Presently, fast and effective searching for relevant images from database becomes an important and challenging investigated topic. Good accuracy and efficiency retrieval solution can not be able to get by using a single feature. It is better to use multi features for image retrieval [9],[12]. A new method using combined features is provided and the experiment is done on the real images. Expected result is achieved by this process and also it shows that combined features are better than the single features for image retrieval, [10], [12].To reach this objective, the pre-processing is first carried out for the improvement of image data which suppresses unwanted distortions and enhances some image features for further processing. Then the feature extraction is carried out. The Feature extraction is a method of constructing combinations of the variables in order to reduce the requirement of large amount of memory and computation power when analysis with a large

DOI : 10.5121/ijcsity.2014.2207 
number of variables present in a data, which describes the data with sufficient accuracy. On this basis, GLCM, SIFT and Moment Invariant features are used for feature extraction. GLCM produce a matrix includes directions and distances between pixels, and it extracts useful statistics from the matrix as a texture features[8],[9]. In computer vision SIFT is an algorithm to detect and describe the local features in images. The interesting points on the object can be extracted to provide a "feature description" of the object of any image [4].Moment invariants are the functions of image moments and the moments are nothing but the projection of the image function into a polynomial basis[3]. Then we retrieve the relevant geographic images from the dataset by using Euclidean distances, [6].

\section{PROPOSED WORK:}

The aim of this project is to retrieve the relevant geographic images from the dataset. The first step is to perform the preprocessing to remove noise by using Gaussian filter. Then the next step is feature extraction. In feature extraction three approaches (i.e.) SIFT, GLCM and Moment invariant are combined. The main purpose for this combination is that if single approach is used, it just extracts the features which are all related to that particular approach alone. So the relevant images only based on that features alone can be retrieved and also the result may not accurate. But, because of combining these three approaches more features can be extracted for better retrieval result. After feature extraction, the relevant images are retrieved from the dataset by using Euclidean distances. The block diagram of our project is given below:

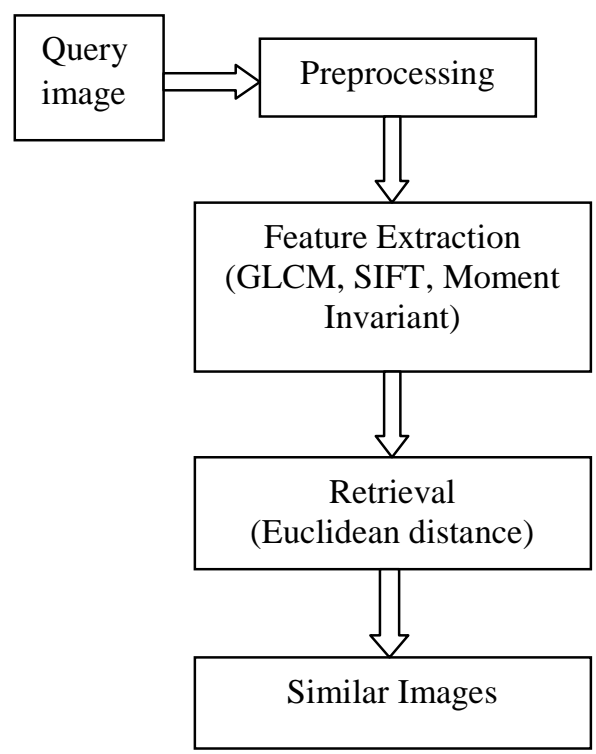

Figure: Block Diagram 
International Journal of Computational Science and Information Technology (IJCSITY) Vol.2, No.2, May 2014

\section{FEATURE EXTRACTION:}

The feature extraction is the important step in this project. Because based on the features extracted from the image, retrieval process has to be done. Here the three feature extraction algorithms are combined. They are Gray Level Concurrence Matrix , Scale Invariant Feature Transform and Moment Invariant features. Let see about the description of these three feature extraction algorithm.

\subsection{SIFT:}

The most powerful method for obtaining the local descriptors is the SIFT(Scale Invariant Feature Transform) [4].It transforms image data into scale-invariant coordinates which is relative to local features. It is based on 4 major stages: scale space extrema detection, localization of key point, assignment of orientation, and key point descriptor, [3]. Let $J(x, y)$ be an image and $L(x, y, \sigma)$ be the scale space of $J$, which is defined as

$$
L(x, y, \sigma)=G(x, y, \sigma) * J(x, y)
$$

where, $*$ is the convolution operation in $x$ and $y$.

$G(x, y, \sigma)$ is a variable-scale Gaussian defined as

$$
G(x, y, \sigma)=\frac{1}{2 \pi \sigma^{2}} e^{-\left(x^{2}+y^{2}\right) / 2 \sigma^{2}}
$$

First the scale-space extrema detection starts with the detection oflocal maxima and minima of $D(x, y, \sigma)$, which is defined as the convolution of a difference of Gaussian with the image $J(x, y)$

$$
\begin{aligned}
D(x, y, \sigma) & =(G(x, y, k \sigma)-G(x, y, \sigma)) * J(x, y) \\
& =L(x, y, k \sigma)-L(x, y, \sigma) .
\end{aligned}
$$

The detection is performed by pointing over all scales and image locations to identify the potential interest points which are invariant to orientation and scale[7]. Once a set of keypoint candidates is obtained, the second step is to accurately localize them. By rejecting the keypoints which have a low contrast or badly localized along an edge, by using a detailed fit to the nearby data for location,scale and ratio of principal curvatures. By taking into account a threshold over the extremum of the Taylor expansion (up to the quadratic terms) of $D(x, y, \sigma)$ the unstable extrema with low contrast will be detected. The third stage is the assignment of orientation to each keypoint, based on local image gradient directions. It allows for the representation of each keypoint which is relative to this orientation, achieving invariance to image rotation. This is performed in the course of an orientation histogram which is formed from the gradient orientations of sample points within a region around the keypoint, which is having 36 bins covering the $360 \circ$ range of orientations. Each sample which gets added to the histogram is weighted by its gradient magnitude and by a Gaussian-weighted circular window with a sigma that is 1-5 times which of the scale of the keypoint[4]. Next, a thresholding-based procedure advanced by a parabola fitting is used to accurately find out the keypoint orientation through the orientation histogram. Final stage is the keypoint descriptor. Last steps assigned the location, scale, and the orientation of each keypoint. The motivation for the estimation of complex 
International Journal of Computational Science and Information Technology (IJCSITY) Vol.2, No.2, May 2014

descriptor is to obtain a highly individual keypoint and invariant as possible to variations such as change in illumination. Each resultant of this SIFT descriptor is a 128-element feature vector .The SIFT approach is little bit slow in detecting the key points, so we first propose Harris corner detector and then apply SIFT descriptor[2]. Harris corner detector removes the low contrast key points . Harris corner detector is a simple point feature extraction algorithm. It has high stability to accurate extraction of corner points even under any circumstance such as rotation, transformation, gray scale, and noise and illumination[2].

\subsection{GLCM:}

The full expansion of GLCM is Gray Level Co-occurance Matrix. It was introduced by Haralick et al[1]. The GLCM is used to extract the fourteen features. They are contrast, correlation, cluster prominence, cluster shade, energy, dissimilarity, entropy, homogeneity, maximum probability, sum of squares, auto correlation, inverse different moment. That is the texture features are extracted according to the statistics of Gray-Level Co-occurance Matrix(GLCM). Here GLCM is used to extract features which are all suitable for geographic image retrieval . Six textural parameters are considered to be the most relavant [1] among the 14 originally proposed in [8][9]: energy, contrast, variance, correlation, entrop and inverse difference moment.It first creates a cooccurance matrix, also called as co-occurance distribution. It is defined over an image to be the distribution of co-occuring values at a given offset. Particularly it represents the distance and angular spatial relationship over an image sub region of specific size. The GLCM is created from a gray scale image. It calculates how often a pixel in an image with gray level value occurs either horizontally or vertically or diagonally to adjacent pixels in an image with the value.

\subsection{MOMENT INVARIANTS:}

Moment Invariants are nothing but the functions of image moments and the mmoments are "projections" of image function into a polynomial basiswhereas the Invariants are functions defined on the image space such that

- $\quad I(f)=I(D(f))$ for all admissible $D$

- $I\left(f_{1}\right), I\left(f_{2}\right)$ "different enough" for different $f_{1}, f_{2}$

The moment invariants are generated based on the information given by the shape boundary and also its interior region,[3]. Theoretically the moments are defined in the continuous but here for practical they are computed in the discrete form,[10]. Bygiving afunction $f(x, y)$, the regular moments are defined by:

$$
\mathrm{M}_{\mathrm{pq}}=\iint x^{\mathrm{p}} \mathrm{y}^{\mathrm{q}}(\mathrm{x}, \mathrm{y}) \mathrm{dx}, \mathrm{dy}
$$

Where, Mpq is the 2-D moment of the function $f(x, y)$.

$(p+q)$ is the order of the momentwhere,$p$ and $q$ are natural numbers. For accomplishment in digital form it becomes:

$$
\mathrm{M}_{\mathrm{pq}}=\sum_{x} * \sum_{y} x^{\mathrm{p}} \mathrm{y}^{\mathrm{q}} \mathrm{f}(\mathrm{x}, \mathrm{y})
$$


The co-ordinates of the centre of gravity of the image are calculated and are given by:

$$
\begin{aligned}
& \bar{x}=\frac{M_{10}}{M_{00}} \\
& \bar{y}=\frac{M_{01}}{M_{00}}
\end{aligned}
$$

The central moments in discrete form is given as:

$$
\mu_{\mathrm{pq}}=\sum_{x} \sum_{y}(x-x)^{\mathrm{p}}(\mathrm{y}-\mathrm{y})^{\mathrm{q}}
$$

For the effects of change in scale the moments are further normalizedby using the formula given below:

$$
\eta_{p, q}=\frac{\mu_{p q}}{\mu_{00}^{\gamma}}
$$

Where the normalisation factor is $\gamma=(\mathrm{p}+\mathrm{q} / 2)+1$.

A set of 7 values can be calculated from the normalised central moments and is given by:

$$
\begin{aligned}
1 & =\eta_{20}+\eta_{02} \\
2 & =\left(\eta_{20}-\eta_{02}\right)^{2}+4 \eta_{11}^{2} \\
3 & =\left(\eta_{30}-3 \eta_{12}\right)^{2}+\left(\eta_{03}-3 \eta_{21}\right)^{2} \\
4 & =\left(\eta_{30}+\eta_{12}\right)^{2}+\left(\eta_{03}+\eta_{21}\right)^{2} \\
5 & =\left(3 \eta_{30}-3 \eta_{12}\right)\left(\eta_{30}+\eta_{12}\right)\left[\left(\eta_{30}+\eta_{12}\right)^{2}-3\left(\eta_{21}+\eta_{03}\right)^{2}\right]+\left(3 \eta_{21}-\eta_{03}\right)\left(\eta_{21}+\eta_{03}\right) \times\left[3\left(\eta_{30}+\eta_{12}\right)^{2}-\left(\eta_{21}+\eta_{03}\right)^{2}\right] \\
6 & =\left(\eta_{20}-\eta_{02}\right)\left[\left(\eta_{30}+\eta_{12}\right)^{2}-\left(\eta_{21}+\eta_{03}\right)^{2}\right]+4 \eta_{11}\left(\eta_{30}+\eta_{12}\right)\left(\eta_{21}+\eta_{03}\right) \\
7 & =\left(3 \eta_{21}-\eta_{03}\right)\left(\eta_{30}+\eta_{12}\right)\left[\left(\eta_{30}+\eta_{12}\right)^{2}-3\left(\eta_{21}+\eta_{03}\right)^{2}\right]+\left(3 \eta_{12}-\eta_{30}\right)\left(\eta_{21}+\eta_{03}\right) \times\left[3\left(\eta_{30}+\eta_{12}\right)^{2}-\left(\eta_{21}+\right.\right. \\
\left.\left.\eta_{30}\right)^{2}\right] &
\end{aligned}
$$

These 7 invariant moments, I, $1 \leq \mathrm{I} \leq 7$, set out by $\mathrm{Hu}$, were as well as shown to be independent of rotation,[10]. However they are computed over the shape boundary and also its interior region.

\section{RETREIVAL:}

Retrieval is the process of collecting the images from the dataset or trainingset which are all related with the image what we are given as the input to the command. Here we are doing retrieval process by using Euclidean distance. The Euclidean distance between two points $\mathrm{x}$ and $\mathrm{y}$ is given as, [6],

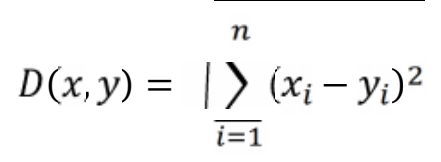

Here we include totally 40 geographic images in the dataset. 


\section{EXPERIMENT:}

In this paper, the training set consists of 40 images with different categories and all are geographical images. Totally it have four categories. First category covers ocean, second category covers cyclone and third category covers images with more roads whereas final category covers building area. Here the objective is to retrieve the relevant images from this. As the first process, the query image is preprocessed by using Gaussian filter in order to remove the noise occurs in the image.

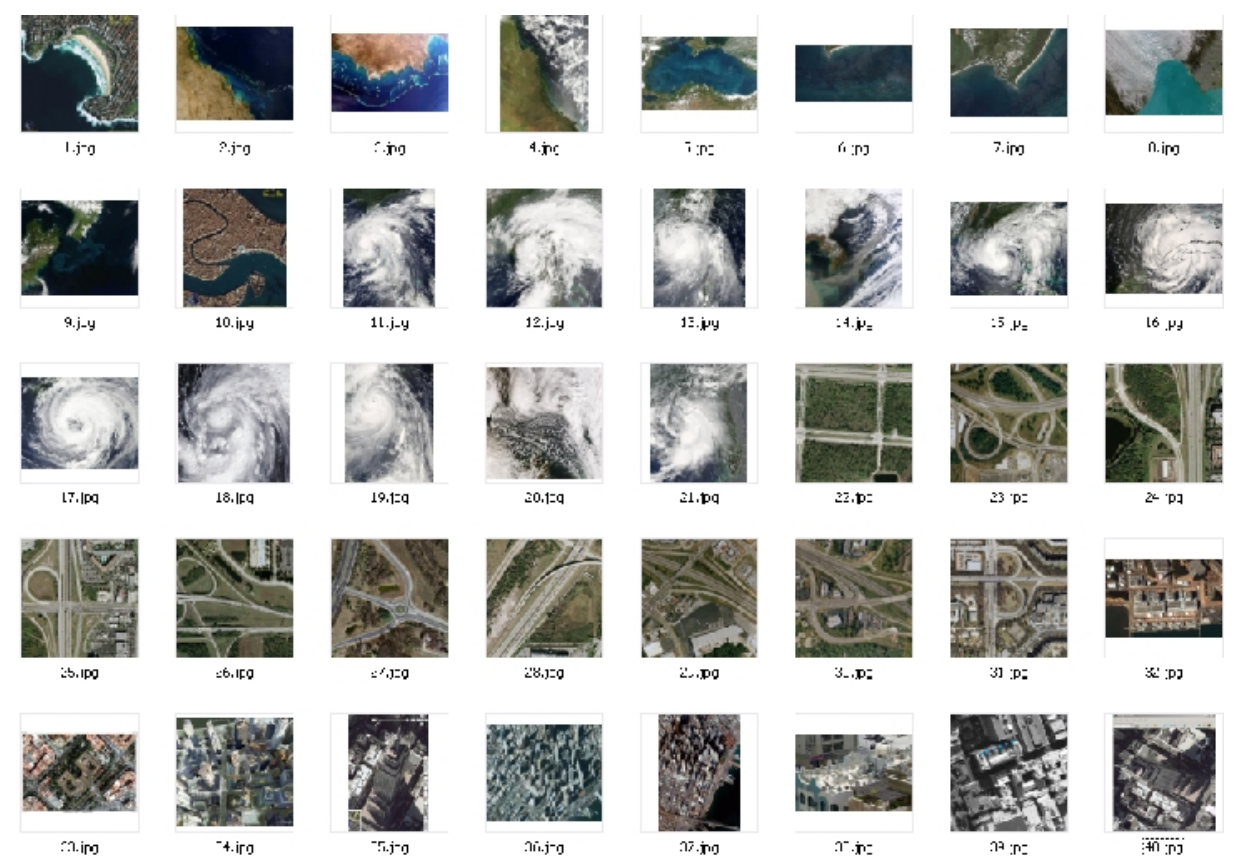

Figure: Dataset which contains totally 40 images. From this dataset only the relevant images are going to retrieve.

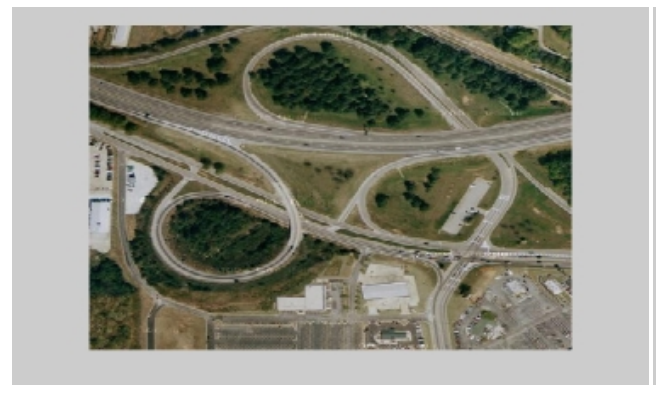

Figure: 1: Input image

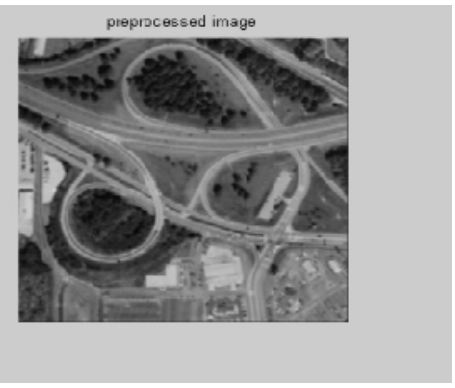

Figure: 2: Preprocessed image 


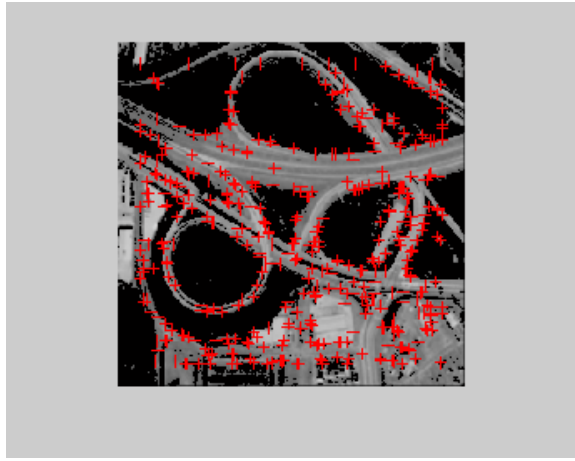

Figure: 3: After finding corners

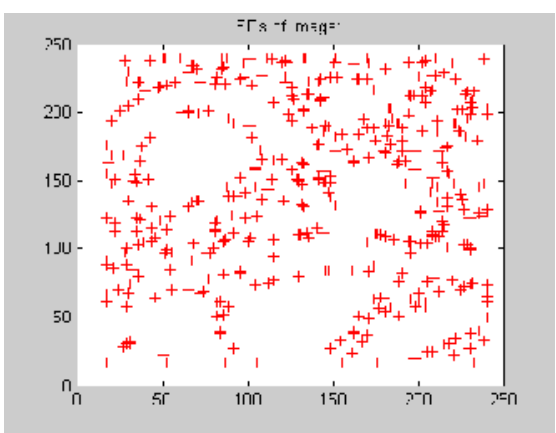

Fig: 4: Plotting of figure 3

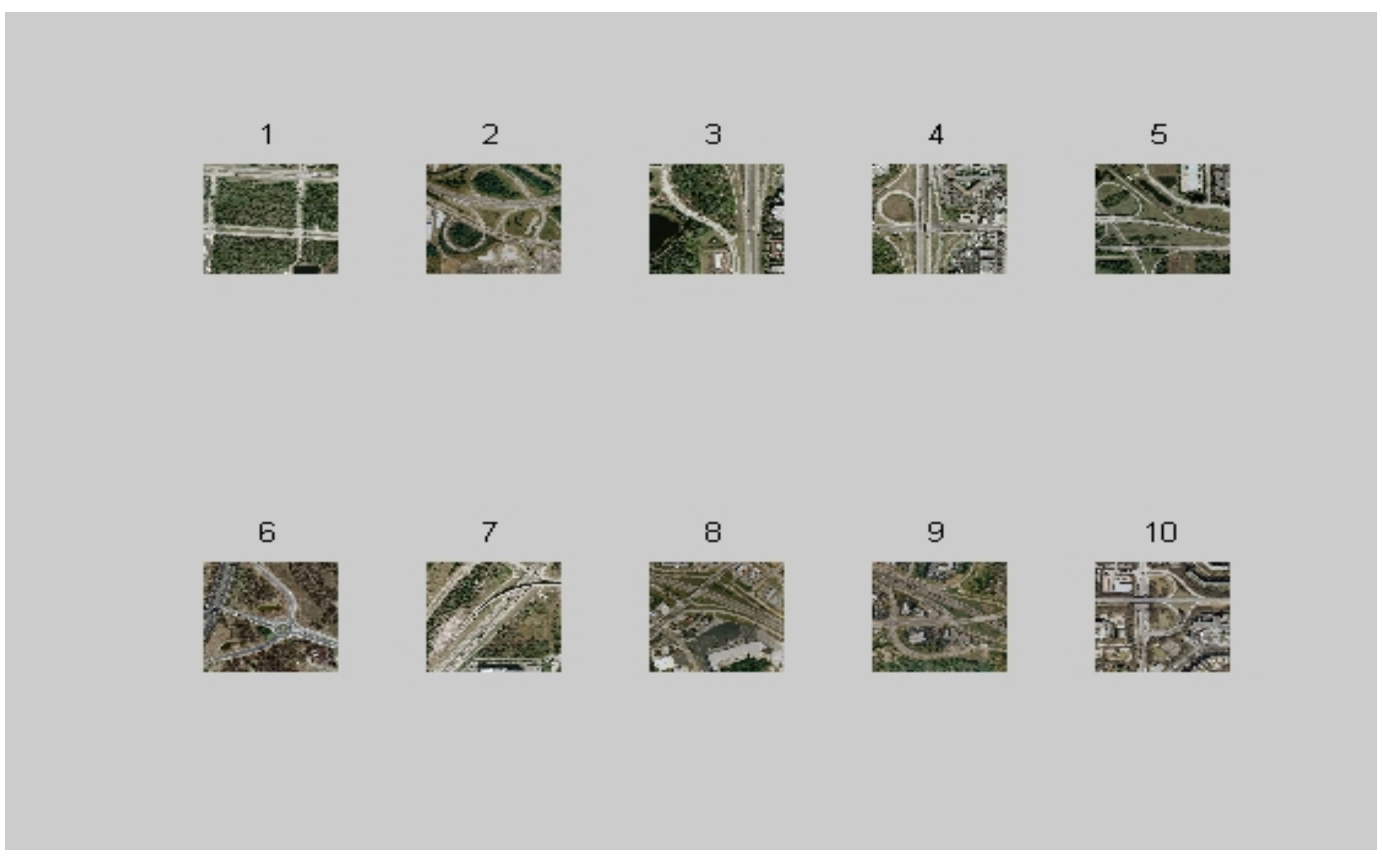

Figure: 5: Retrieved output which shows related images to the input image.

The figure one shows the query image on which basis the retrieval output is going to get. Before starts the process all the images in the training set and also the query images get resized into 256 X 256 resolutions. The figure two shows the preprocessed query image. It has done with help of Gaussian filter.. The second step of this project is feature extraction. In this three approaches are combined. They are GLCM, SIFT and Moment invariant. In GLCM approaches, it creates the cooccurrence matrix also called as gray-co matrix. It first initially assigns zero value to matrix. After the first iteration step, it assigns the values in the matrix. Finally it fills the full values in that matrix. The GLCM is used to extract six features [1]. They are energy, contrast, variance, correlation, entropy and inverse difference moment. The values of each get stored in each variables starting from v1 to v12. Before the SIFT approach is applied, Harris corner detector is applied to extract the corner points very accurately even under any circumstance such as rotation, translation, gray scale, noise and illumination [2]. The figure three shows the result of after finding corners. It accurately shows the corners which have high contrast. In SIFT, first the 
extrema is calculated for the scale-space. Then the key points are localized. The nearby points are interpolated. The low contrast key points and the edge responses are eliminated. The shape features are extracted by using moment invariant [7]. By using the moment invariant we extract totally seven features and all results are values,[3]. All the three get added into the single variable in mat lab coding. This represents the combined of three approaches. After this process, the same method applied to the query image will be applied to each image in the training set. The figure 5 shows the retrieved output. As the input image contains more roads, the retrieved images also contain the images which contain more roads. It is retrieved by using the Euclidean distance algorithm. Based on the features extracted values the relevant images get retrieved.
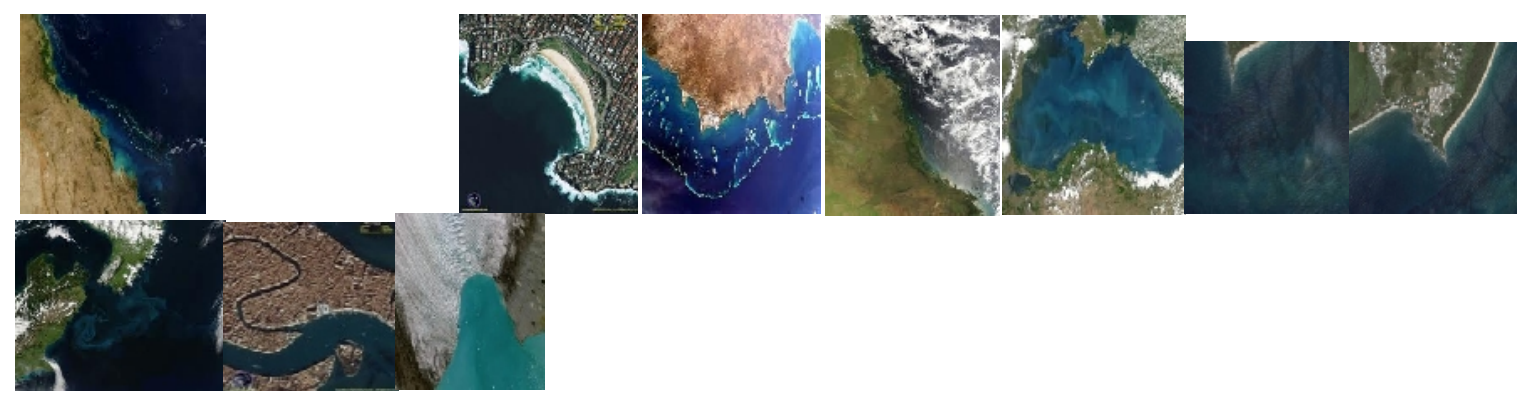

Fig: (a)- The input image and corresponded retrieved ocean output

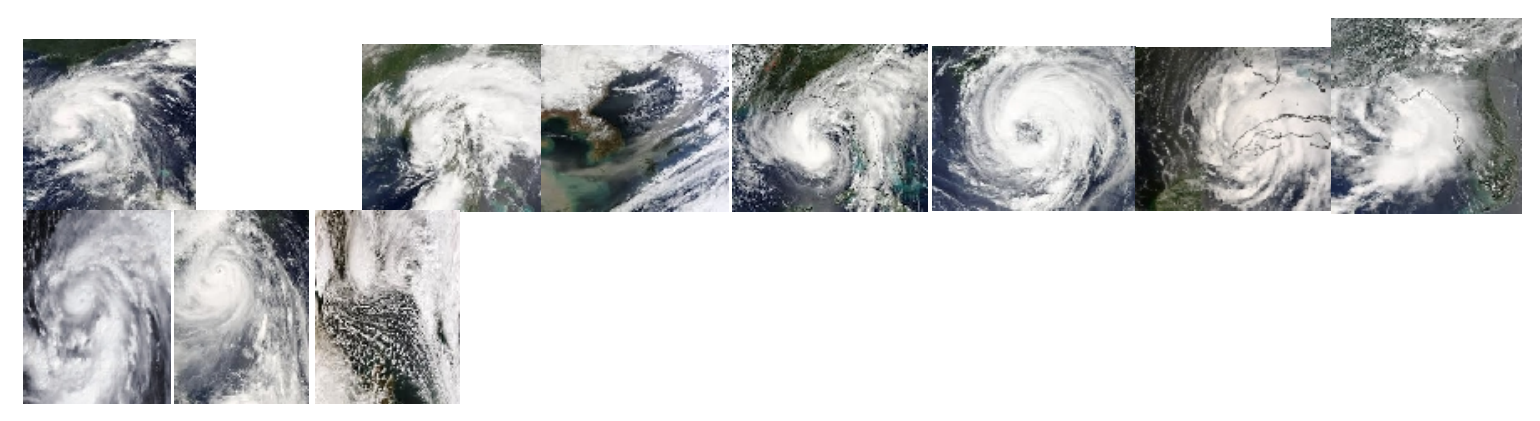

Fig: (b) - The input image and respected retrieved cyclone output

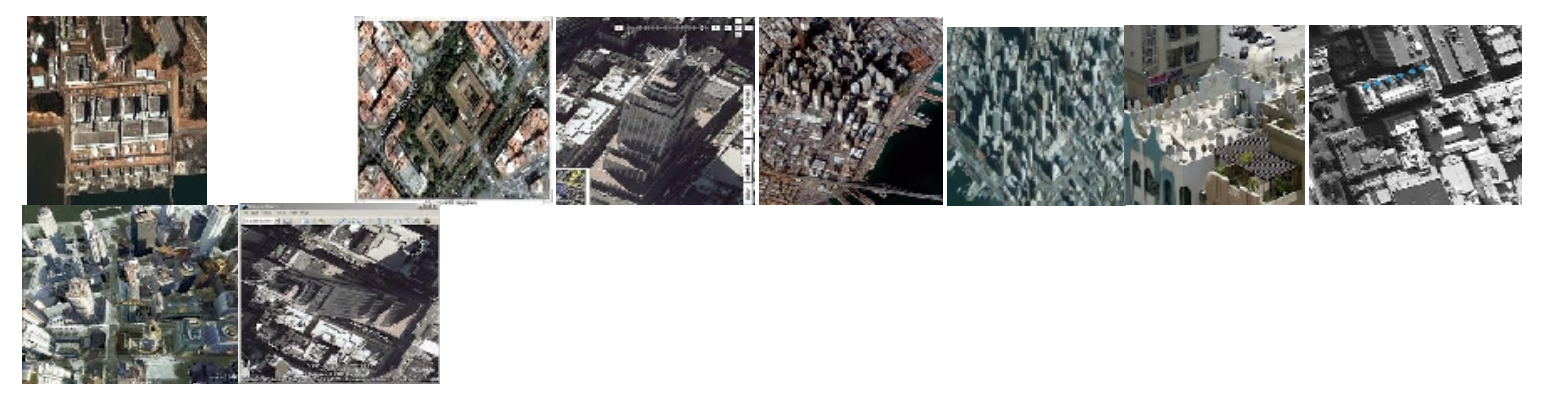

Fig: (c)- The input image and respected retrieved building output

In figure a, the input image is ocean and the corresponded ocean images are retrieved as the output. Likewise in figure $b$, we are giving cyclone image as the input and so the respected 
cyclone images are retrieved from the training set. Similarly in figure c, the image with buildings is given as input and the relevant images are retrieved from the training set respectively.

\section{CONCLUSION:}

This paper presents an approach for geographic image retrieval. The retrieved output shows that the combination of three feature extraction technique (Gray Level Concurrence Matrix, Scale Invariant Feature Transform and Moment Invariant) yields an accurate retrieval result. Because more features are extracted to provide the better retrieval result. This project is used in remote sensing, to locate the similar locations.

\section{REFERENCES:}

[1] Andre Baraldi and Flavio Panniggiani, "An Investigation of the Textural Characteristics Associated with Gray Level Cooccurence Matrix Statistical Parameters", IEEE TRANSACTIONS ON GEOSCIENCE AND REMOTE SENSING, VOL. 33, NO. 2, MARCH 1995293.

[2] J.Dai, W.Song, L.Pei, and J.Zhang, "Remote sensing image matchng via Harris detector and SIFT descriptor", in Proc.Int.Congr,Image Signal Process, 2010,vol.5,pp.2221-2224.

[3] K.Mikolajczyk and C.Schmid, "A performance evaluation of local descriptors," IEEE Trans. Pattern Anal. Mach. Intell, vol. 27, no. 10, pp.1615-1630, oct.2005.

[4] H.Goncalves, L.Corte-Real, and J.Goncalves, "Automatic image registration through image segmentation and SIFT, “IEEE Trans.Geosci, Remote Sens, vol. 49, no.7, pp. 2589-2600, Jul.2011.

[5] S.Belongie, J.Malik, and J.Puzicha, "Shape matching and object recognition using shape contents, "IEEE Trans. Pattern Anal. Mach. Intell., vol. 24, no. 4, pp. 509-522, Apr. 2002.

[6] Jer-Sheng Chou: Tung Shou Chen, "An efficient computation of Euclidean distances using approximated look-up table”, IEEE, Transc on Circuits and Systems for video Technology, vol,10 isssue-4.

[7] Y. Ke and R. Sukthankar, "PCA-SIFT: A more distinctive representation for local image descriptors," in Proc. IEEE Int. Conf. Comput. Vis. Pattern Recog., 2004, vol. 2, pp. 506-513.

[8] Fan-Hui Kong, "Image Retrieval Using Both Color And Texture Features", Int.Conf on Machine Learning and Cybemtics, Baoding, 12-15 July-2009.

[9] R.M.Haralick, K.Shanmugam and I.Dinstein, "Textural features for image classification, “ IEEE Trans.Systems, Manufact Cybernet, vol SMC-3 no.6 pp 610-621 2010.

[10] R.J.Ramteke,'Invarient Moments Based Feature Extraction for Hand written Devanagari vowels recognition",International Journal of Computer Applications, vol1-no.18.

[11] Kebin Jia, Zhuozheng Wang, Zhuoyi Lv, "A New and Effective Image Retrieval Method Based on Combined Features", Int.conf on Image and Graphics, 2007.

[12] Lefei Zhang, Liangpei Zhang, Dacheng Tao, Xin Huang, "On Combining Multiple Features for Hyperspectral Remote Sensing Image Classification”, IEEE Trans. On Geoscience and Remote sensing on vol 50 , issue: 3 . 